\title{
EFEITOS FISIOLÓGICOS DO TREINAMENTO RESISTIDO NO CONTROLE HEMODINÂMICO
}

DOI: $10.48140 /$ digitaleditora.2020.002.18

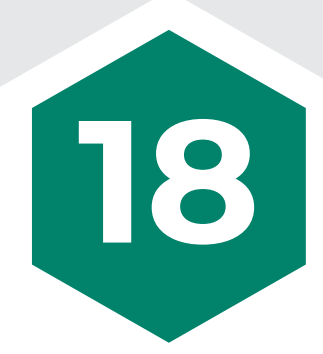

RESUMO

Objetivos: Descrever os benefícios da utilização do treinamento resistido para a realização do controle hemodinâmico, identificando quais efeitos fisiológicos são gerados pelo treinamento resistido no controle da hipertensão e da hipotensão arterial

Métodos: Trata-se de um estudo sobre como o treinamento resistido contribui para a existência de reações fisiológicas que corroborem com o tratamento da hipertensão e da hipotensão arterial, o mesmo foi realizado através de buscas em artigos de revistas especializadas e nacionais, disponíveis nas bases de dados referentes ao período entre 2010 a 2020. Portanto, trata-se de uma revisão integrativa através de uma pesquisa bibliográfica, ou seja, uma revisão da literatura sobre as principais teorias que norteiam o trabalho científico.

Resultados: Percebe-se que os autores convergem ao afirmarem que o treino resistido (TR) pode promover melhorias às pessoas com acometimento cardiovasculares. Porém, o que mais observou-se foi o bom resultado perante o trabaIho com pré-hipertensos e com hipertensos. Para realizar a redução da Pressão Arterial Sistólica o mais adequado seria a utilização de sessões de 3 circuitos. Já para a Pressão Arterial Diastólica o mais adequado seria ou sessões de 1 circuito, ou sessões de 3 circuitos ou 3 séries convencionais. Portanto, percebe-se que uma série de 3 circuitos se adequa aos dois tipos de comorbidade.

Conclusão: Conclui-se que apesar do Treino Resistido promover uma elevação da frequência cardíaca e da pressão arterial, este quando utilizado de forma monitorada e adaptado aos indivíduos que têm acometimento cardiovascular torna-se um treino extremamente eficaz e uma boa possibilidade de treino adaptado aos hipertensos/hipotensos, pois além de propiciar saúde física, levando bem estar para o indivíduo e um melhor condicionamento físico, também gera a existência de mais resistência da pessoa acometida, inclusive para lidar com situações diárias como subir uma escada, fazer um passeio mais longo caminhando, carregar um determi-

Cícero Eduardo Mota

Mesquita da Silva

Graduando em Educação Física pela AESPI - Ensino Superior do Piauí Teresina - Piauí

https://orcid

iD org/0000-0002 $3247-0619$

Tâmyack Alves de Macêdo

Graduando em Educação Física pela AESPI - Ensino Superior do Piauí

Teresina - Piauí

https://orcid

iD org/0000-0002 $4110-9729$

Tâmyack Alves de Macêdo

Profissional de Educação Física, Especialista Professor da Faculdade AESPI - Ensino Superior do Piauí - Teresina - Piauí https://orcid

iD org/0000-0002 8583-8325 nado peso, dentre outras possibilidades.

PALAVRAS-CHAVES: treino resistido. exercício físico. problemas cardiovasculares. hipertensão arterial. hipotensão arterial. 


\title{
PHYSIOLOGICAL EFFECTS OF RESISTED TRAINING IN HEMODYNAMIC CONTROL
}

\author{
DOI: $10.48140 /$ digitaleditora.2020.002.18
}

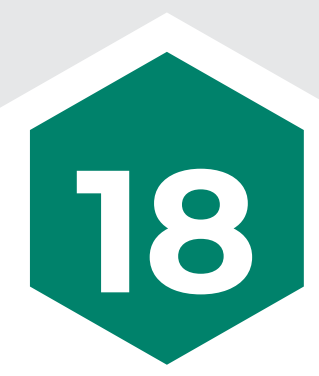

Recebido em: 10/12/2020 Aprovado em: 28/12/2020 Conflito de Interesse: não Suporte Financeiro: não houve

\section{ABSTRACT}

Objectives: To describe the benefits of using resistance training to carry out hypertension and hypotension control, identifying which physiological effects are generated by resistance training in the control of hypertension and hypotension. Methods: This is a study on how resistance training contributes to the existence of physiological reactions that corroborate the treatment of hypertension and hypotension, the same was done through searches in articles of specialized and national magazines, available in the databases of data for the period from 2010 to 2017. Therefore, it will be a systematic review through a literature search, that is, a review of the literature on the main theories that guide scientific work.

Results: It is noticed that the authors converge when they affirm that the resistance training (RT) can promote improvements to the people with cardiovascular involvement. However, what was most observed was the good result when working with pre-hypertensive and hypertensive patients. To perform the reduction of systolic blood pressure, the most appropriate would be the use of 3 circuit sessions. For Diastolic Blood Pressure, the most suitable would be either 1 circuit sessions, or 3 circuit sessions or 3 conventional series. Therefore, it is clear that a series of 3 circuits is suitable for both types of comorbidity.

Conclusion: It is concluded that, although Resistance Training promotes an increase in heart rate and blood pressure, when used in a monitored way and adapted to individuals who have cardiovascular involvement, it becomes an extremely effective training and a good possibility of adapted training. hypertensive / hypotensive patients, because in addition to providing physical health, bringing well-being to the individual and better physical conditioning, it also provides the existence of more resistance of the person affected even to deal with daily situations such as climbing a ladder, taking a longer walk, carrying a certain weight, among other possibilities. 


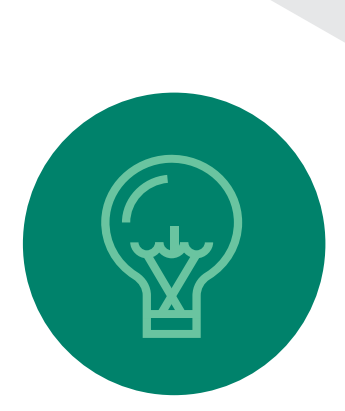

\section{INTRODUÇÃO}

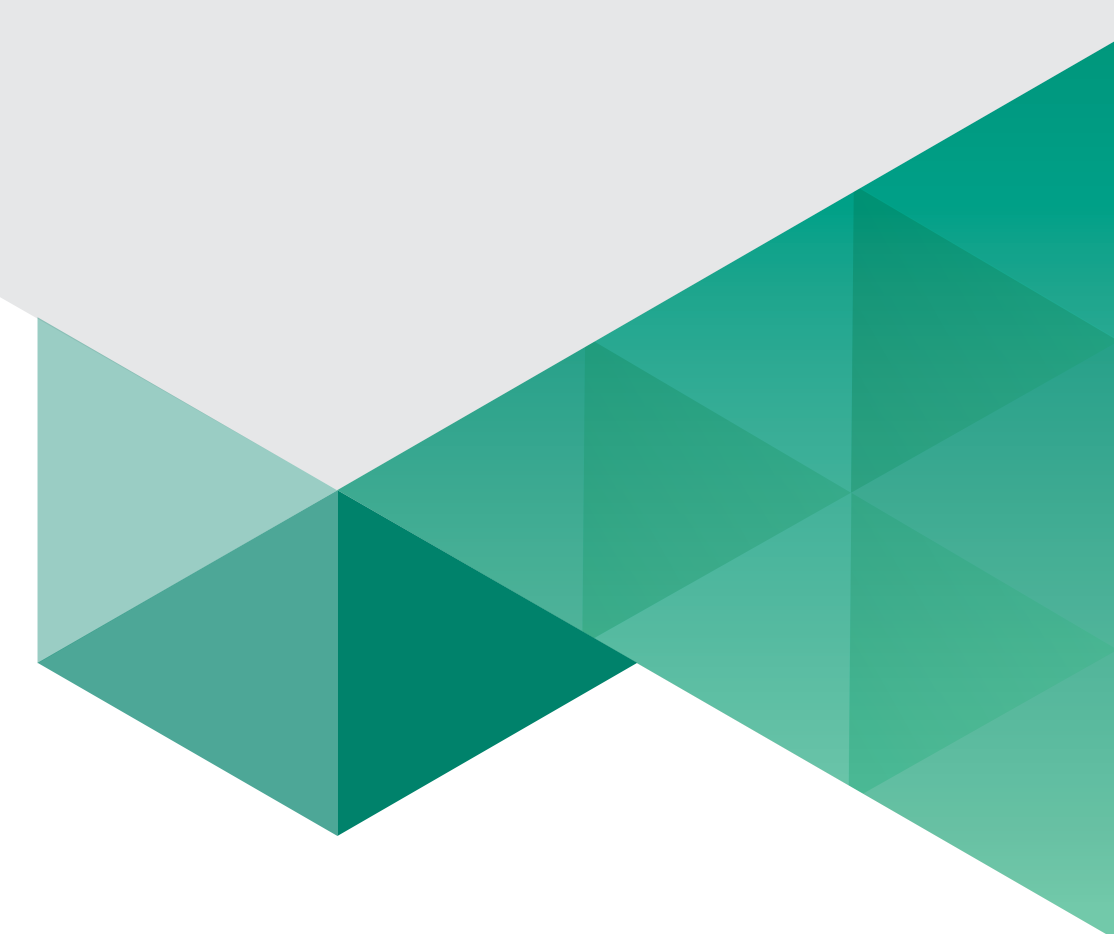

Compreendem-se como exercícios resistidos aqueles em que há contração muscular de um segmento corporal contra uma força que se opõe (MEDINA et al., 2010). Os exercícios resistidos são inicialmente destinados ao ganho de força, resistência e potência muscular, através de exercícios que variam em relação ao número de repetições, de séries e do tempo de repouso entre elas, normalmente realizados contra resistência gerada por equipamentos ou pelo próprio corpo (SAUNDERS et al., 2013).

A PAS (Pressão arterial sistólica) aumenta drasticamente durante o exercício resistido e a relevância da resposta hipertensiva depende da intensidade do esforço realizado e da quantidade de massa muscular atuante, desse modo, intensidades de esforço com elevadas frequências devem ser desencorajadas para indivíduos com doenças cardiovasculares, sobretudo para os destreinados, pois poderia ser prejudicial. Contudo, esse tipo de exercício poderá ser praticado por esse grupo a partir da intensidade baixa até a moderada, a hipotensão pós-exercício pode ter efeito de até 12 horas após uma sessão de treinamento resistido (MCARDLE, KATCH \& KATCH, 2016).

A Sociedade Brasileira de Cardiologia - SBC (2010, p.1) define a hipertensão arterial sistêmica (HAS) como uma condição clínica multifatorial caracterizada por níveis elevados e sustentados de pressão arterial. No Brasil, as Doenças Cardiovasculares têm sido a principal causa de morte.

A Hipertensão Arterial Sistêmica apresenta grande impacto na saúde pública devido à sua alta taxa de incidência, que chega a atingir 26\% da população mundial e $68 \%$ dos idosos, e, também, por representar um dos principais fatores de risco para o desenvolvimento de doenças (LIM et al., 2012). Tal condição consiste em uma condição clínica multifatorial caracterizada por elevados e sustentados níveis de pressão arterial (PA) e classificada de acordo com os valores pressóricos encontrados: entre 121/81 e 139/89 - pré-hipertensão, entre 140/90 e 159/99 mmHg - hipertensão estágio I, entre 160/100 e 179/109 - estágio II, e, por fim, $\geq 180 / 110$ - estágio III. Para os casos em que os valores de PA sistólica (PAS) e a PA diastólica (PAD) estejam situados em categorias diferentes a classificação é feita de acordo com o maior valor (MALACHIAS et al., 2016).

Uma das alternativas para o controle da HAS é o treinamento resistido (TR), o qual é realizado através de contrações voluntárias da musculatura esquelética contra determinada forma de resistência, que pode ser o próprio peso corporal isolado ou associado a pesos externos ou equipamentos dentro de uma sessão de treino (FLECK \& KRAEMER, 2017). 
No entanto, são conhecidas as evidências de que a PA pode sofrer expressivos aumentos durante o Exercício Resistido (ER), sobretudo quando executado até a fadiga, sem, todavia, causar danos ao sistema cardiovascular (DUTRA et al., 2012). Durante a realização do ER, a PA aumenta não somente por conta da intensidade do exercício, mas também devido à duração, atingindo seus picos nas últimas repetições e próximo à falha concêntrica, na qual se observa o aumento do componente isométrico.

Estudos de meta-análise têm demonstrado que a prática de exercícios resistidos pode contribuir para o tratamento e/ou prevenção de disfunções cardiovasculares como a HAS (CUNHA et al., 2012). Observa-se que nos estudos sobre treinamento resistido para hipertensos, as intensidades utilizadas variam de leve a pesada, não havendo um consenso acerca da intensidade ideal para a redução dos níveis tensionais (NETO, FILHO \& FARINATTI, 2010). Além disso, são escassas as informações sobre a influência do intervalo de recuperação entre séries de exercícios (MEDINA et al., 2010).

Desse modo, fica evidente à necessidade de obter estudos que tragam planos de treino eficazes no tratamento da hipertensão e da hipotensão arterial, possibilitando gerar benefícios como o desenvolvimento de potência, força e resistência muscular, a diminuição de gordura corporal, e o aumento de massa magra, fatores estes que favorecem a existência de uma melhor aptidão física e proporciona qualidade de vida por facilitar a realização de atividades cotidianas, como carregar peso ou subir escadas (KALIDE \& RODRIGUES, 2015).

Dessa forma, o presente estudo visa, através de uma revisão integrativa, buscar na literatura resultados da utilização do treinamento resistido para a realização do controle da hipertensão e da hipotensão arterial, identificando quais efeitos fisiológicos são gerados pela prática dessa modalidade de treinamento.

\section{METODOLOGIA}

TEste artigo trata-se de uma pesquisa de revisão integrativa sobre como o treinamento resistido contribui para a existência de reações fisiológicas que corroborem com o controle hemodinâmico. O desenvolvimento da pesquisa foi elaborado a partir de materiais já existentes e encontrados em artigos científicos, sendo embasado nas fontes bibliográficas, no qual se refere a exploração dos fatos relatados em cada artigo.

As publicações foram coletadas nas seguintes bases de dados: Scientific Eletronic library online (SCIELO), Literatura Latina-Americano e de Caribe em Ciências da Saúde (LILACS), Public Medicine (PubMed), Red de Revistas Científicas de América Latina e el Caribe (REDALYC), Repositório Institucional da Universidade Federal de Uberlândia (UFU), Public Knowledge Project (PKP) e outros. Foram utilizadas as seguintes palavras-chaves: treino resistido, exercício físico, problemas vasculares, hipertensão e hipotensão arterial em inglês e português entre os anos de 2010 a 2020. As palavras foram cruzadas utilizando o conectivo "and".

Foram incluídos, na pesquisa, artigos na íntegra que tratavam da seguinte temática: efeitos fisiológicos do treinamento resistido no controle da hipertensão e da hipotensão arterial, nas bases de dados referidas acima, nos idiomas português e inglês e publicados no período de 2010 a 2020. Foram excluídos artigos publicados 
fora do tempo limite, artigos de revisão, que não são relacionados a humanos e que fujam do padrão a ser exposto no trabalho.

Os dados foram organizados utilizando o programa Microsoft Word 2010, na pesquisa foram levadas em consideração as questões éticas, assegurando a autoria dos artigos pesquisados. Não houve a necessidade de aprovação do comitê de ética, pois no trabalho não foi realizado estudos com humanos e nem com animais.

\section{RESULTADOS}

Somando-se todas as bases de dados, foram encontrados 81 artigos. Após a leitura dos títulos dos artigos, notou-se que alguns deles se repetiram nas diferentes bases e outros não preenchiam os critérios desde estudo.

Quadro 1 - Publicações disponíveis no período de 2010 à 2017, aplicando as palavras chaves treino resistido, exercício físico, problemas vasculares, hipertensão e hipotensão arterial nas 3 bases de dados (SCIELO, LILACS, PubMed, REDALYC, UFU, PKP e outros).

\begin{tabular}{|l|c|c|c|c|}
\hline $\begin{array}{l}\text { BASES DE } \\
\text { DADOS }\end{array}$ & $\begin{array}{c}\text { TREINO RESISTIDO } \\
\text { AND PROBLEMAS } \\
\text { VASCULARES }\end{array}$ & $\begin{array}{c}\text { TREINO RESISTIDO } \\
\text { AND HIPERTENSÃO } \\
\text { AND HIPOTENSÃO } \\
\text { ARTERIAL }\end{array}$ & $\begin{array}{c}\text { EXERCÍCIO FÍSICO AND } \\
\text { HIPERTENÇÃO AND HIPO- } \\
\text { TENÇÃO ARTERIAL }\end{array}$ & Total \\
\hline SCIELO & 4 & 1 & 1 & 6 \\
\hline LILACS & 1 & 1 & & 2 \\
\hline PUbMed & & 1 & 1 & 1 \\
\hline REDALYC & & 1 & & 2 \\
\hline UFU & & 1 & & 1 \\
\hline PKP & & 1 & 1 & 2 \\
\hline Outros & & 2 & 3 & 1 \\
\hline TOTAL & 5 & 7 & & 15 \\
\hline
\end{tabular}


Quadro 2 - Publicações disponíveis no período de 2010 à 2017, aplicando as palavras chaves treino resistido, exercício físico, problemas vasculares, hipertensão e hipotensão arterial nas 3 bases de dados (SCIELO, LILACS, PubMed, REDALYC, UFU, PKP e outros).

\begin{tabular}{|c|c|}
\hline Autor (ano) & Principais achados \\
\hline $\begin{array}{l}\text { ANUNCIAÇÃO, P. G. et al., } \\
\text { (2012) }\end{array}$ & $\begin{array}{l}\text { Verificar as respostas cardiovasculares após o exercício resistido realizado de } \\
\text { diferentes formas e volumes de trabalho. }\end{array}$ \\
\hline $\begin{array}{l}\text { ARAÚJO, J. M. M. M. et al., } \\
\text { (2020) }\end{array}$ & $\begin{array}{l}\text { Foi realizado uma revisão da literatura onde foram analisados artigos pu- } \\
\text { blicados entre os anos de } 2014 \text { a } 2019 \text {. Os estudos coletados utilizaram } 8 \\
\text { ou mais semanas de intervenção e cuja investigação envolveu a análise das } \\
\text { respostas pressóricas em mmHg. Os resultados revelaram, após o processo } \\
\text { de análise, que o TR obteve redução semelhantes dos níveis pressóricos aos } \\
\text { obtidos no TC, sem diferença significativa para ambos os métodos. }\end{array}$ \\
\hline BENTO, & $\begin{array}{l}\text { Avaliar o impacto de intervenções } \\
\text { de brasileiros. Meta-análise feita pc } \\
\text { várias bases de dados em ciências }\end{array}$ \\
\hline $\begin{array}{l}\text { BORGE } \\
\text { D.; \& D }\end{array}$ & $\begin{array}{l}\text { e analisaram os efeitos agudos e } \\
\text { sistido }\end{array}$ \\
\hline S. da et al., (2012) & amento resistido sobre a pres- \\
\hline DUTR & à Hipotensão Pós-Exercício \\
\hline LOCKS, & $\begin{array}{l}\text { eróbio e resistido nas res- } \\
\text { s de treinamento promo- } \\
\text { uso. }\end{array}$ \\
\hline MEDIN/ & $\begin{array}{l}\text { a sobre a pressão } \\
\text { ra sua prescrição. }\end{array}$ \\
\hline $\begin{array}{l}\text { NETO, A. G. C.; FILHO, I. R. } \\
\text { da C. \& FARINATTI, P. T. V., } \\
\text { (2010) }\end{array}$ & $\begin{array}{l}\text { Investigar a influência do núme } \\
\text { los de recuperação entre séries } \\
\text { arterial sistólica (PAS) e duplo p }\end{array}$ \\
\hline POTON \& & $\begin{array}{l}\text { as durante o exercí- } \\
\text { sanguíneo). }\end{array}$ \\
\hline $\begin{array}{l}\text { PRADO, } \\
(2012)\end{array}$ & ealizar uma revisão teórica acerca do efeito hipotensor do exercício físico. \\
\hline REIS, J. P. C. dos e & $\begin{array}{l}\text { sessões de } 50 \% \text { e } 75 \% \\
\text { u } 1 .\end{array}$ \\
\hline $\mathrm{ROCHA}$, & $\begin{array}{l}\text { Investigar na literatura as alterações cardiovasculares agudas, subagudas e } \\
\text { crônicas diante do exercício resistido. O ER pode gerar alterações agudas, } \\
\text { subagudas e crônicas na pressão arterial PA. }\end{array}$ \\
\hline SOUSA, E. C. d & $\begin{array}{l}\text { Avaliar os efeitos do treinamento resistido sobre a pressão arterial sistólica e } \\
\text { a diastólica em indivíduos pré-hipertensos e hipertensos. }\end{array}$ \\
\hline . & $\begin{array}{l}\text { O estudo consistiu em uma pesquisa de intervenção, com abordagem quan- } \\
\text { titativa de cunho descritivo. }\end{array}$ \\
\hline
\end{tabular}

Fonte: Autoria própria, 2020. 


\section{DISCUSSÃO}

É evidente a necessidade de estudos sobre treinamento resistido e seus efeitos fisiológicos no controle da hipertensão e hipotensão arterial. Diante disso, faz-se necessário a existência dessa atualização. Desse modo, esta será realizada contando com a literatura como importante fonte para responder a questionamentos.

No Brasil, as doenças cardiovasculares são a principal causa de morte, gerando custos médicos e socioeconômicos bastante elevados. A hipertensão arterial sistêmica (HAS) é um fator de risco altamente prevalente em nosso meio, responsável por aproximadamente $45 \%$ dos casos de doenças cardíacas isquêmicas, e 51\% das doenças cerebrovasculares. As modificações do estilo de vida, em especial atividade física e modificações dietéticas são a base do tratamento do paciente hipertenso, em nível primário e secundário (BENTO et al., 2015).

A prevalência da hipertensão arterial sistêmica (HAS) tem crescido consideravelmente em diversos países do mundo, diversos estudos têm indicado que a prática de exercícios físicos regulares pode provocar modificações importantes na PA, tanto em indivíduos normotensos como em hipertensos. Estudos de meta-análise têm demonstrado que a prática de exercícios resistidos pode contribuir para o tratamento e/ou prevenção de disfunções cardiovasculares como a HAS (CUNHA et al.,2012). Seguindo nessa mesma linha, Locks et al., (2012) têm descrito que tanto o exercício aeróbio quanto o treinamento resistido, quando realizados isoladamente, promovem benefícios imediatos e em longo prazo, como redução da pressão arterial em repouso, melhora da capacidade cardiorrespiratória e atenuação das respostas cardiovasculares ao esforço. Levando-se em consideração que poucos são os estudos que avaliam os efeitos da associação de exercícios aeróbios e resistidos.

Segundo Medina et al. (2012) a prática regular de atividades físicas de lazer, principalmente vigorosas, reduz em aproximadamente 30\% o risco de desenvolvimento da HA. O treinamento aeróbico reduz a PA clínica sistólica/diastólica de hipertensos em cerca de $7 / 5 \mathrm{mmHg}$, além de diminuir a PA de vigília e em situações de estresse físico e mental. Por outro lado, o treinamento resistido não parece reduzir substancialmente a PA de hipertensos, embora traga outros benefícios à saúde.

Observando diversos estudos, Anunciação et al., (2012) relataram queda na pressão arterial (PA) após uma única sessão de exercício, fenômeno este que vem sendo denominado de hipotensão pós-exercício (HPE). O exercício aeróbio parece ser o mais eficiente para promover a queda da PA pós-esforço. Em contrapartida, em relação ao exercício resistido há menor quantidade de informações disponíveis na literatura. Apesar de os resultados obtidos nos estudos envolvendo o exercício resistido sugerirem que este modelo de exercício é capaz de promover a HPE, algumas vezes os resultados dessas investigações são contraditórios.

São conhecidas as evidências de que a PA pode sofrer expressivos aumentos durante o Exercício Resistido (ER), sobretudo quando executado até a fadiga, sem, no entanto, causar danos ao sistema cardiovascular. Ademais, a prática do ER tem sido recomendada para reduzir o risco de acometimentos cardiovasculares tanto em normotensos como em hipertensos (DUTRA et al., 2013).

Durante a realização do ER, a PA aumenta não somente por conta da intensidade do exercício, mas também devido à duração, atingindo seus picos nas últimas repetições e próximo à falha concêntrica, na qual se observa o aumento do componente isométrico (ROCHA et al., 2012). Além disso, Nery et al., (2010) evidenciaram resposta pressórica exacerbada em sujeitos com hipertensão, quando comparado com normotensos, independentemente da carga (40\% ou 80\% da carga máxima dinâmica) utilizada durante o exercício de cadeira extensora até a falha concêntrica. Esses dados reforçam a importância 
da carga relativa no comportamento da PA, como também a resposta mais acentuada em hipertensos.

A PAS (Pressão arterial sistólica) aumenta drasticamente durante o exercício resistido e, a magnitude da resposta hipertensiva depende da intensidade do esforço realizado e da quantidade de massa muscular atuante, desse modo, intensidades de esforço elevadas devem ser desencorajadas para indivíduos com doença cardíaca e vascular, sobretudo para os destreinados, pois poderia ser prejudicial. Contudo, esse tipo de exercício poderá ser praticado por esse grupo a partir da intensidade baixa até a moderada, a hipotensão pós- exercício pode ter efeito de ate 12 horas após uma sessão de treinamento resistido (VÉRAS et al.,2020).

Reis et al., (2015) mostraram que o treinamento resistido (TR) possibilita a redução da pressão arterial pós-treino, hipotensão pós-exercício (HPE) em indivíduos normotensos e principalmente em hipertensos, no entanto existem divergências quando se especificam as conclusões da influência da intensidade nessa resposta em indivíduos hipertensos. Durante o TR a resposta das variáveis hemodinâmicas, frequência cardíaca ( $F C$ ), pressão arterial sistólica (PAS) e diastólica (PAD) são dependentes das variáveis dessa modalidade de treino: carga, volume do treino, tempo de tensão, massa muscular mobilizada, intervalo entre séries e intervalo entre exercícios.

Araújo et al., (2020) demonstraram algumas evidências que a prática de TR ao longo de algumas semanas e com frequência semanal de 3 sessões semanais tem se mostrado eficaz na diminuição crônicas da pressão arterial sistólica (PAS) e diastólica (PAD) de repouso. Em outros estudos os resultados também revelaram reduções na PAS e na PAD em idosos após programas de TR. Nesse sentido, a utilização do TR como uma estratégia terapêutica não medicamentosa para o tratamento de HAS tem se mostrado eficiente em programas de exercícios para indivíduos jovens e idosos hipertensos.

Neto et al., (2010) compararam informações sobre a influência do intervalo de recuperação entre séries e exercícios. Apenas um estudo investigou especificamente a influência de tal variável sobre os valores de FC e PA, identificando maior resposta pressórica em sessões com intervalos mais curtos. Todavia, o aumento progressivo da pulsação ao longo das séries foi mais evidente para intervalos mais curtos. Vale ressaltar que esses estudos aplicaram intervalos de recuperação fixos. Assim, não consideraram o efeito cumulativo da fadiga muscular no decorrer das séries. Na prática, percebe-se que diferentes exercícios necessitam de tempo de execução variável, seja pela complexidade ou pela amplitude requerida do movimento.

Poton e Polito, (2014) relataram que o exercício resistido é indicado como estratégia para o aumento da força muscular. Para isso, a intensidade do esforço deve estar entre 60-85\% de uma repetição máxima (1RM)3 . Entretanto estudos recentes 4-11 mostram que o exercício resistido realizado em intensidades menores que 50\% 1RM com restrição de fluxo sanguíneo (RFS) tem se mostrado eficiente em promover modificações morfológicas e fisiológicas semelhantes aos exercícios com intensidades mais elevadas sem RFS. o comportamento da pressão arterial (PA) durante o exercício tem relação direta com a carga. 


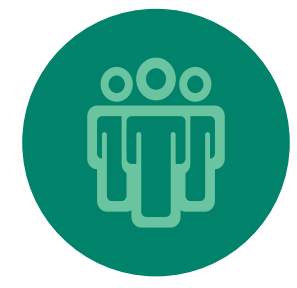

\section{CONCLUSÃO}

Conclui-se que a realização desta revisão permitiu identificar diferentes olhares dos pesquisadores sobre objeto de estudo discutido os efeitos do treinamento resistido para a realização do controle da hipertensão e hipotensão arterial. Pode-se concluir que vários autores convergem ao afirmarem que o treino resistido (TR) pode promover melhorias às pessoas com acometimento cardiovasculares. Os resultados demonstram achados que podem ter aplicações importantes para a avaliação e prescrição mais adequadas de programas de exercícios. 


\section{REFERÊNCIAS}

ANUNCIAÇÃO, P. G.; PORTON, R.; SZYTKO, A.; POLITO, M. D. Comportamento cardiovascular após o exercício resistido realizado de diferentes formas e volumes de trabalho. Rev. Bras. Med. Esporte, Vol.18, № 2, Mar/Abr, 2012. Disponível em: <https://www.scielo.br/scielo.php?script=sci_arttext\&pi$d=S 1517-86922012000200011>$. Acesso em: 12/09/2020.

ARAÚJO, J. M. M. M.; COSTA, E. M. da; SILVA, E. R. F. da; ROCHA, R. W. G. da; FARIAS, A. M. de. Efeitos do treinamento resistido e do treinamento combinado sobre os níveis pressóricos de portadores de hipertensão arterial sistêmica. Braz. J.Hea. Ver., Curitiba, v.3, n.4, p.7081-7089,, jul/ago. 2020. Disponível em: <https://www.brazilianjournals.com/index.php/BJHR/article/view/13039>. Acesso em: 04/12/2020.

BENTO, V. F. R.; ALBINO, F. B.; MOURA, K. F. de; MAFTUM, G. J.; SANTOS, M. de C. dos; GUARITA-SOUZA, L. C.; NETO, J. R. F.; BAENA, C. P. Impacto de intervenções em atividade física sobre a pressão arterial de populações brasileiras. Arq. Bras. Cardiol., 105(3): 301-308, 2015. Disponível em: <https:// www.scielo.br/scielo.php?script=sci_arttext\&pid=S0066-782X2015002200301\&lang=pt>. Acesso em: 04/12/2020.

BORGES, D. X.; NUNES, J. E. D.; \& DOURADO, V. Z. O efeito do treinamento resistido na hipertensao arterial: revisão sistemática. UNIFESP-SP, 2014. Disponível em: <https://repositorio.ufu.br/bitstream/123456789/24608/3/EfeitoTreinamentoResistido.pdf>. Acesso em: 12/09/2020.

CUNHA, E. S. da; MIRANDA, P. A. de; NOGUEIRA, S.; COSTA, E. C.; SILVA, E. P. da; FERREIRA, G. M. H. Intensidades de treinamento resistido e pressão arterial de idosas hipertensas- um estudo piloto. Ver. Bras. de Med. do Esporte, vol.18, n066, São Paulo - SP, nov/dez, 2012. Disponível em: <https://www. scielo.br/scielo.php?script=sci_arttext\&pid=S1517-86922012000600005\&lng=pt\&nrm=iso>. Acesso em: 10/09/2020.

DUTRA, M. T.; LIMA, R. M.; MOTA, M. R.; OLIVEIRA, P. F. A. de; VELOSO, J. H. C. L. Hipotensão pós exercício resistido: uma revisão da literatura. Rev. Educ. Fis/UEM, v. 24, n. 01, p. 145-157, 1. trim., 2013. Disponível em: <https://www.scielo.br/pdf/refuem/v24n1/15.pdf>. Acesso em: 12/09/2020.

FLECK, S. J.; KRAEMER, W. J. Fundamentos do treinamento de força muscular. 4oth ed. [place unknown]: Artmed Editora; 2017.

KALIDE, L. S.; RODRIGUES, N. A. O Treinamento Resistido e os Benefícios Fisiológicos, Psicológicos e Sociais na Vida dos Idosos. Universidade do Vale do Paraíba(UNIVAP)- Faculdade de Educação e Artes curso de Educação Física, São José dos Campos - SP, 2015. Disponível em: <https://biblioteca.univap. br/dados/000021/000021cd.pdf>. Acesso em: 10/09/2020.

LIM, S. S.; VOS, T.; FLAXMAN, A. D.; DANAEI, G.; SHIBUYA, K.; ADAIR-ROHANI, H., et al. A comparative risk assessment of burden of disease and injury attributable to 67 risk factors and risk factor clusters in 21 regions, 1990-2010: A systematic analysis for the Global Burden of Disease Study 2010. Lancet. 2012;380(9859):2224-60.

LOCKS, R. R.; RIBAS, D. I. R.; WACHHOLZ, P. A.; GOMES, A. R. S. Efeitos do treinamento aeróbico resistido nas respostas cardiovasculares de idosos ativos. Fisioter. Mov., Curitiba, v.25, n.3, p.541-550, jul/ set, 2012. Disponível em:<https://www.scielo.br/pdf/fm/v25n3/10.pdf> Acesso em: 03/12/2020. 
MALACHIAS, M. V. B.; PLAVNIK, F. L.; MACHADO, C. A.; MALTA, D.; L. C. N. SCALA. 7ạ Diretriz Brasileira de Hipertensão Arterial: Capítulo 1- Conceituação, Epidemiologia e Prevenção Primária. Arquivos Brasileiros de Cardiologia, 2016.

MCARDLE, W. D.; KATCH, F. I.; KATCH, V. L. Fisiologia do exercício. Nutrição, energia e desempenho humano. 8. Ed. Rio de Janeiro: Guanabara Koogan, 2016.

MEDINA, F. L.; LOBO, F. da S.; SOUZA, D. R. de; KANEGUSUKU, H.; FORJAZ, C. L. de M. Atividade física: impacto sobre a pressão arterial. Rev. Bras. Hipertens., vol.17(2): 103-106, 2010. Disponível em: <http://cev.org.br/arquivo/biblioteca/4030390.pdf>. Acesso em: 03/12/2020.

NETO, A. G. C.; FILHO, I. R. da C. \& FARINATTI, P. T. V. Respostas Cardiovasculares ao Exercício Resistido são Afetadas pela Carga e Intervalos entre Séries. Sociedade Brasileira de Cardiologia, Universidade Salgado de Oliveira, Niterói - RJ, 2010. Disponível em: <https://www.scielo.br/pdf/abc/2010nahead/ aop11510.pdf>. Acesso em: 10/09/2020.

POTON, R.; POLITO, M. D. Respostas Cardiovasculares durante exercício resistido com restrição de fluxo sanguíneo. Rev. Bras. Cardiol. 27(2): 104-110, 2014. Disponível em: <http://bases.bireme.br/cgi-bin/wxislind.exe/iah/online/? IsisScript=iah/iah.xis\&src=google\&base=LILACS\&lang=p\&nextAction=Ink\&exprSearch=719582\&indexSearch=|D>. Acesso em: 03/12/2020.

PRADO, A. L. M.; SILVA, C. E. B.; BRITO, C. P.; PRADO, M. C. M.; PRADO, O. M. P. Exercício físico no tratamento da hipertensão arterial sistêmica: Hipotensão pós-exercício e prescrição de exercício físico para pacientes hipertensos. Motricidade, vol. 8, núm. Supl. 2, 2012, pp. 719-724 Desafio Singular-Unipessoal, Lda Vila Real, Portugal. Disponível em: <https://www.redalyc.org/pdf/2730/273023568085.pdf>. Acesso em: 14/09/2020.

REIS, J. P. C. dos; ALMEIDA, K. da S.; SOUZA, R. A. da S.; SOUSA, M. R. S. S. de. Efeito do treinamento resistido com diferentes intensidades na pressão arterial em hipertensos. Internacional Journal of Cardiovascular Sciences. 2015;28(1):25-34. Disponível em: <http://www.onlineijcs.org/english/sumario/28/pdf/v28n1a05.pdf>. Acesso em: 14/09/2020.

ROCHA, A. C.; MORAES-SILVA, I. C.; QUINTEIRO, R. G.; SARTORI, M.; ANGELIS, K. D. Ajustes agudos, subagudos e crônicos da pressão arterial ao exercício resistido. ConScientiae Saúde, 11(4):685-690, 2012. Disponível em: <https://www.redalyc.org/pdf/929/92924959020.pdf>. Acesso em: 03/12/2020.

SAUNDERS, D.; SANDERSON, M.; BRAZZELLI, M.; GREIG, C.; MEAD, G. Physical fitness training for stroke patients. Cochrane Database of Systematic Reviews. 2013. <https://doi.org/10.1002/14651858. CD003316.pub5>. Acesso em: 04/12/2020.

SOCIEDADE BRASILEIRA DE CARDIOLOGIA. VI Diretrizes Brasileiras de Hipertensão Arterial. Ver. Bras. Hipertens; 2010; 17(1): 1-64.

SOUSA, E. C. de; ABRAHIN, O.; FERREIRA, A. L. L.; RODRIGUES, R. P.; ALVES, E. A. C.; VIERA, R. P. Resistance training alone reduces systolic and diastolic blood pressure in prehypertensive and hypertensive individuals: meta-analysis. The Japanese Society of Hypertension All rights reserved 0916-9636/17, 2017. Disponível em: <https://pubmed.ncbi.nlm.nih.gov/28769100/>. Acesso em: 14/09/2020.

VÉRAS, M. L. V.; RODRIGUES, J. D.; CAETANO, E. C. de S.; SILVA, V. G. da; NASCIMENTO, R. A. Comportamento da pressão arterial de idoso hipertenso antes e após sessão de treinamento resistido: um estudo de caso. Braz. J. of Develop., Curitiba, v.6, n.4, p.21420-21428, abr. 2020. Disponível em: <https:// www.brazilianjournals.com/index.php/BRJD/article/view/9250>. Acesso em: 04/12/2020. 